\title{
miR-146a targeted to splenic macrophages prevents sepsis-induced multiple organ injury
}

\author{
Yoshio Funahashi $\mathbb{D}^{1,2} \cdot$ Noritoshi Kato $^{2} \cdot$ Tomohiro Masuda $^{2} \cdot$ Fumitoshi Nishio $^{3} \cdot$ Hiroki Kitai $^{1,2} \cdot$ Takuji Ishimoto $^{2}$. \\ Tomoki Kosugi $^{2} \cdot{\text { Naotake Tsuboi } \mathbb{D}^{2}}^{2}$ Naoyuki Matsuda ${ }^{4} \cdot$ Shoichi Maruyama ${ }^{2} \cdot$ Kenji Kadomatsu $^{1}$
}

Received: 22 August 2018 / Revised: 28 November 2018 / Accepted: 13 December 2018 / Published online: 30 January 2019

(c) United States \& Canadian Academy of Pathology 2019

\begin{abstract}
Development of a novel agent against life-threatening sepsis requires the in-depth understanding of the relevant pathophysiology and therapeutic targets. Given the function of microRNAs (miRNAs) as potent oligonucleotide therapeutics, here we investigated the pathophysiological role of exogenously applied miRNA in sepsis-induced multiple organ injury. In vitro, miR-16, miR-126, miR-146a, and miR-200b suppressed the production of pro-inflammatory cytokines in RAW264.7 macrophage cells after lipopolysaccharide (LPS) stimulation. Of these, miR-146a displayed the most highly suppressive effect, wherein the transcriptional activity of nuclear factor kappa B (NF- $\mathrm{KB}$ ) was decreased via targeting of interleukin 1 receptor-associated kinase 1 and tumor necrosis receptor-associated factor 6 . Sepsis was induced in mice via cecal ligation and puncture (CLP) and an intravenous injection of a complex of miR-146a-expressing plasmid and polyethyleneimine. Treatment with this complex significantly decreased the level of serum inflammatory cytokines, attenuated organ injury including kidney injury, and led to increased survival from polymicrobial sepsis induced by CLP. miR-146a-expressing plasmid was abundantly distributed in splenic macrophages, but not in renal parenchymal cells. CLP mice treated with miR-146a displayed significantly decreased NF- $\mathrm{KB}$ activation and splenocyte apoptosis. Splenectomy diminished the anti-inflammatory effects of miR-146a. The collective results support the conclusion that the induction of miR-146a expression in splenic macrophages prevents excessive inflammation and sepsis-induced multiple organ injury. This study establishes a novel and critical pathophysiological role for splenic macrophage interference in sepsis-related organ injury.
\end{abstract}

Supplementary information The online version of this article (https:// doi.org/10.1038/s41374-019-0190-4) contains supplementary material, which is available to authorized users.

$\triangle$ Noritoshi Kato

n-kato@med.nagoya-u.ac.jp

1 Department of Biochemistry, Nagoya University Graduate School of Medicine. 65 Tsurumai-cho, Showa-ku, Nagoya, Aichi, Japan

2 Department of Nephrology, Nagoya University Graduate School of Medicine, 65 Tsurumai-cho, Showa-ku, Nagoya, Aichi, Japan

3 Department of Nephrology, Tsushima City Hospital. 3-73, Tachibana-cho, Tsushima, Aichi, Japan

4 Department of Emergency and Critical Care Medicine, Nagoya University Graduate School of Medicine, 65 Tsurumai-cho, Showa-ku, Nagoya, Aichi, Japan

\section{Introduction}

Sepsis is a life-threatening organ dysfunction caused by an uncontrolled host response to infection. Sepsis is a major cause of morbidity and mortality in the intensive care unit (ICU) [1]. Sepsis-related mortality rates are high in patients with multiple organ failure [2]. Prior to the onset of sepsis, bacteria or bacterial products penetrate external barriers, such as the epithelium. The next line of defense is the reticuloendothelial system comprising the liver, lymph node, bone marrow, and spleen, which is pivotal in innate immunity. The response of the host defense system to bacterial invasion results in the immediate activation of innate immunity, but an excessive immune response may cause a hyper-inflammatory cytokine storm, multiple organ failure, and even death [3, 4]. The Toll-like receptor (TLR)/nuclear factor kappa B (NF- $\mathrm{KB}$ ) system is crucial in the pathogenesis of sepsis. TLR-mediated signaling leads to the formation of a complex between interleukin (IL)-1R-associated kinase 4 
(IRAK-4) and tumor necrosis factor receptor-associated factor 6 (TRAF6), which leads to the phosphorylation of inhibitory $\kappa-\mathrm{B}(\mathrm{I} \kappa \mathrm{B})$. This reaction facilitates the NF- $\kappa \mathrm{B}-$ mediated activation of the transcription of pro-inflammatory mediators in the nucleus [5-8]. In septic conditions, dysregulated NF- $\mathrm{NB}$ activity induces a cytokine storm, which results in multiple organ failure [9].

MicroRNAs (miRNAs) are non-coding RNAs that are approximately 20-30 nucleotides in length. They bind to the $3^{\prime}$ untranslated region of target mRNAs, resulting in translational repression or mRNA degradation [10]. Animal studies and clinical trials have demonstrated that the modulation of miRNA expression may be beneficial in several diseases [1113]. Thus, miRNAs have attracted a great deal of attention as a new class of oligonucleotide-based therapeutics. miR-146a is induced by $\mathrm{NF}-\kappa \mathrm{B}$ and acts as a negative feedback regulator of the pro-inflammatory response through the inhibition of TRAF6 and IRAK-1/2 [14-17].

Here, we investigated the pathophysiological role of exogenously applied miR-146a in the attenuation of sepsisinduced hyper-inflammatory states and multiple organ injury. The results demonstrate that the spleen, particularly splenic macrophages, governs the hierarchy of organs in the pathogenesis of sepsis, suggesting that the spleen may be the ideal target in the treatment of sepsis.

\section{Methods}

\section{miRNA reagents and miRNA-expressing plasmid}

MiRNA mimics and a randomly sequenced miRNA negative control were purchased from Life Technologies (Carlsbad, CA, USA). The sequences are described in Supplemental Table 1. miRNA- and enhanced green fluorescent protein (EGFP)-expressing plasmid was purchased from Cell Biolabs, Inc. (San Diego, CA, USA). The miRNA precursor and empty (control) plasmid sequences are listed in Supplemental Table 2A and B.

\section{Animal model of sepsis}

This study was approved by the Institutional Animal Care and Use Committee of Nagoya University School of Medicine (protocol number 29342) and was performed in accordance with the animal experimentation guidelines of Nagoya University School of Medicine. Male C57BL/6 mice, 10-12-weeks old (Chubu Kagaku Shizai, Nagoya, Japan) were subjected to polymicrobial sepsis via cecal ligation and puncture (CLP) surgery as previously described (Fig. S1) [18]. Briefly, under isoflurane anesthesia, a 5-mm incision was made in the left abdomen. The cecum was exposed, ligated $10 \mathrm{~mm}$ from the tip, and punctured once with a 21-gauge needle, followed by gentle squeezing to remove the cecal contents. The abdominal incision was closed in two layers using 5-0 silk sutures. Sham-operated mice were subjected to the same surgical laparotomy, but the cecum was not ligated or punctured. Survival was assessed every $6 \mathrm{~h}$ after surgery, and blood samples were collected $24 \mathrm{~h}$ post-surgery by puncture of the inferior vena cava. Organs were fixed using standard methods [19].

\section{In vitro delivery of miRNA}

RAW264.7 macrophage cells were cultured in six-well plates using low glucose Dulbecco's modified Eagle's medium (Takara Bio, Shiga, Japan) containing 10\% fetal bovine serum (Life Technologies) at $37{ }^{\circ} \mathrm{C}$ in an atmosphere of $5 \% \mathrm{CO}_{2}$. Cells were transfected with miR-146a mimics $(50 \mathrm{nM})$ or a negative control using Lipofectamine 2000 reagent (Invitrogen, Tokyo, Japan). Cells were stimulated for $24 \mathrm{~h}$ post-transfection with $1 \mathrm{mg} / \mathrm{mL}$ of lipopolysaccharide (LPS) (Sigma-Aldrich, Tokyo, Japan) for $30 \mathrm{~min}$ for the examination of NF- $\kappa \mathrm{B}$ transcriptional activity, or $6 \mathrm{~h}$ for the assessment of cytokine production and analysis of IRAK-1 and TRAF6 levels.

\section{In vivo delivery of plasmid DNA}

For plasmid delivery, linear polyethyleneimine (PEI) nanoparticles (in vivo-jetPEI ${ }^{\circledR}$ ) purchased from Polyplustransfection SA (Illkirch-Graffenstaden, France) were used. Plasmid/PEI complex was prepared as previously described and injected into mice via the tail vein 7 days before surgery [20].

\section{Ex vivo imaging}

Mice were sacrificed 7 days after the plasmid injection. Fresh organs were collected and placed in the IVIS Spectrum in vivo imaging system (PerkinElmer, Waltham, MA, USA). EGFP was excited at $465 \mathrm{~nm}$ and detected at $520 \mathrm{~nm}$ and data were collected as photon $\cdot \mathrm{sec} \cdot{ }^{-1} \mathrm{~cm}^{-2}$.

\section{Histological analysis}

Organs were fixed in $4 \%$ paraformaldehyde, embedded in paraffin, cut into $4 \mu \mathrm{m}$ thick sections, and stained with periodic acid-Schiff reagent [21]. Renal tubular injury was evaluated as tubular epithelial swelling, brush border loss, vacuolar degeneration, tubule necrosis, cast formation, and desquamation. The degree of kidney damage was estimated at $400 \times$ magnification using five randomly selected fields for each animal [22]. 
Immunostaining was performed using antibodies against cleaved caspase-3 (Cell Signaling Technology, Danvers, MA, USA), Ly6B (Bio-Rad, Hercules, CA, USA), EGFP (Abcam, Cambridge, MA, USA), neutrophil gelatinaseassociated lipocalin (NGAL; Abcam), and CD68 (Bio-Rad) according to the manufacturers' protocols. Histological evaluations were performed by two blinded observers. TdTmediated dUTP nick and label (TUNEL) staining was performed using an in situ Apoptosis Detection Kit (Takara Bio) according to the manufacturer's protocol.

\section{Blood biochemical analyses}

Blood urea nitrogen (BUN), creatinine (Cre), aspartate aminotransferase (AST), alanine aminotransferase (ALT), and lactate dehydrogenase (LDH) levels in serum were measured using an automated chemistry analyzer (LSI Medience, Tokyo, Japan).

\section{Reverse transcription polymerase chain reaction (RT-PCR)}

Target miRNA was reverse-transcribed using a TaqMan MicroRNA Reverse Transcription Kit (Applied Biosystems, Carlsbad, CA, USA). Target genes were amplified by realtime PCR with a Step One Plus Real-Time PCR System (Applied Biosystems) with all samples assayed in duplicate. Quantitative evaluation of target gene expression was performed using the $\Delta \Delta \mathrm{CT}$ method. mRNA expression was normalized to $\beta$-actin expression, while miRNA expression was normalized to U6 snRNA expression. Primer sequences can be provided upon request.

\section{Transcriptional activity assay}

The activity of NF- $\kappa \mathrm{B}$ was quantified using a TransAM NF$\kappa \mathrm{B}$ p65 Transcription Factor Assay Kit (Active Motif, Carlsbad, CA, USA), according to the manufacturer's recommendation. Optical density (OD) values for the peroxidase reaction product from each sample were normalized to those of $2.5 \mu \mathrm{g}$ of Jurkat nuclear extract provided as a positive control. The activity of activator protein-1 (AP-1) was quantified using a TransAM AP-1 Transcription Factor Assay Kit (Active Motif), according to the manufacturer's recommendation. OD values for the peroxidase reaction product from each sample were normalized to those of $5 \mu \mathrm{g}$ of K-562 nuclear extract provided as a positive control.

\section{Enzyme-linked immunosorbent assay (ELISA)-based cytokine assay}

Levels of cytokines (tumor necrosis factor-alpha [TNF- $\alpha$ ], IL-6, and monocyte chemoattractant protein 1 [MCP-1]) were measured using a Quantikine ELISA Kit (R\&D Systems, Minneapolis, MN, USA) and all samples were assayed in duplicate.

\section{Western blot analysis}

Western blot analysis was performed as previously described [21]. The primary antibodies used were rabbit antimouse IRAK-1 (sc-7883; Santa Cruz Biotechnology, Dallas, TX), TRAF6 antibody (sc-7221, Santa Cruz Biotechnology), or mouse anti- $\beta$-actin antibody (Sigma-Aldrich).

\section{Statistics}

Data are expressed as mean \pm standard error (SE) and significant differences with respect to the interactive effects were analyzed using one-way ANOVA and Tukey's test, respectively. Statistical significance between the two groups was determined by a two-tailed Student's $t$-test. The logrank test was used to compare group survival trends. A $p$ value $<0.05$ was considered statistically significant. GraphPad Prism ver.8 (GraphPad Software, San Diego, CA, USA) was used for all statistical analyses.

\section{Results}

\section{Monocytes overexpressing miR-146a are tolerant of the LPS-induced inflammatory response}

We tested the anti-inflammatory effects of miR-16, miR126, miR-146a, and miR-200b, which are all considered negative regulators of the TLR/NF- $\kappa B$ pathway (Fig. 1a) [23-26]. RAW264.7 cells were transfected with the miRNA mimics or a negative control and then treated with $1 \mathrm{mg} / \mathrm{mL}$ of LPS for $6 \mathrm{~h}$. miR-146a displayed the highest suppressive effect among all the miRNAs tested as evident by the decreased TNF- $\alpha$ and IL- 6 production (Fig. 1b, c).

\section{miR-146a decreases transcriptional activity of NF-KB and AP-1 by targeting IRAK-1 and TRAF6}

Next, we explored the role of miR-146a in the regulation of the TLR/NF- $\mathrm{kB}$ pathway. Enhancement in miR-146a expression resulted in the negative regulation of the expression of IRAK-1 and TRAF6 in RAW264.7 cells (Fig. 1d-f). In addition, we evaluated the activity of NF- $\mathrm{KB}$ and AP-1 after treatment with $1 \mathrm{mg} / \mathrm{mL}$ LPS and found that miR-146a significantly attenuated $\mathrm{NF}-\mathrm{\kappa B}$ and AP-1 activity as compared to the negative control (Fig. 1g, h). The data indicated that miR-146a inhibits LPS-induced inflammatory response by targeting IRAK-1 and TRAF6. 

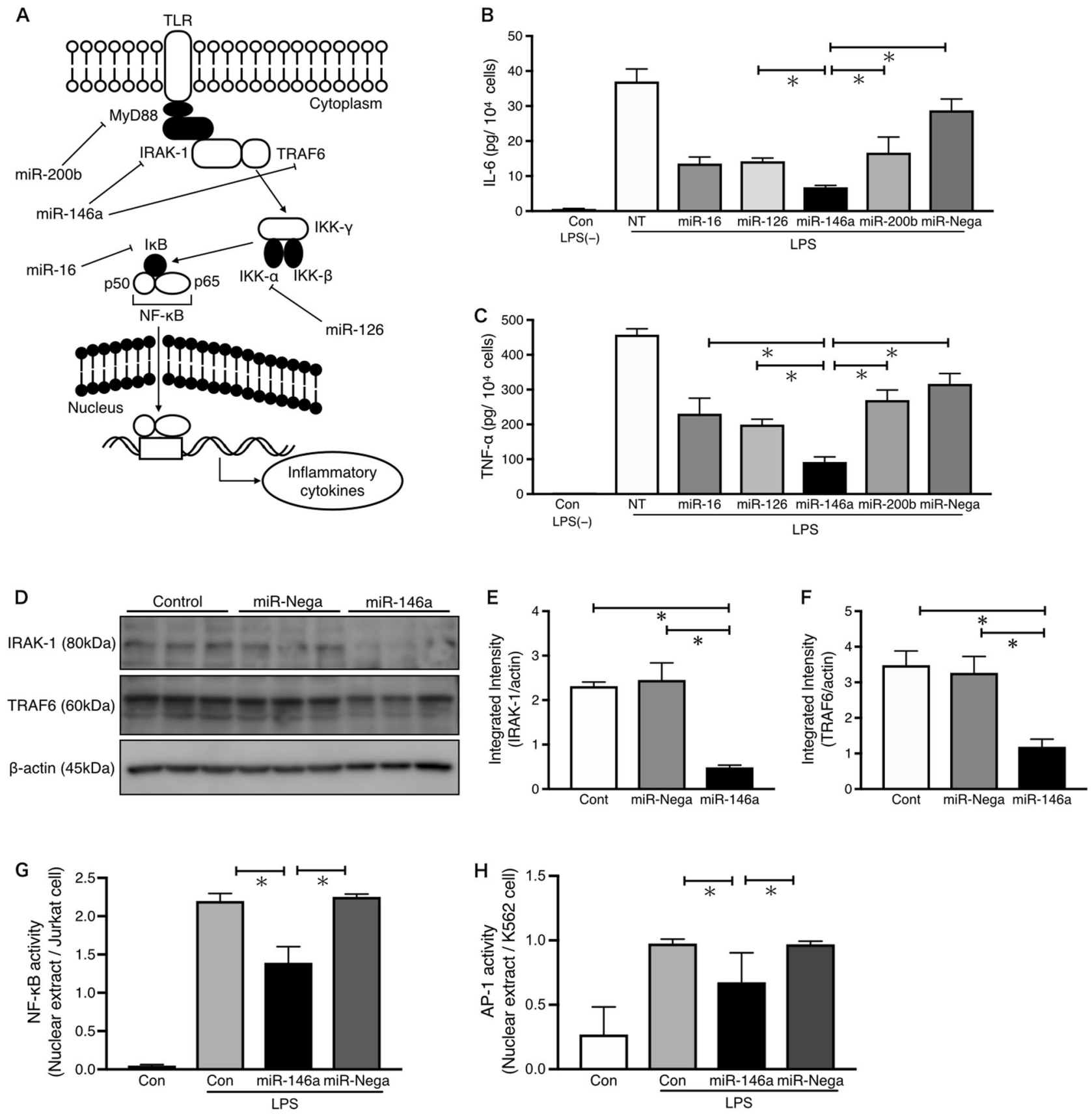

Fig. 1 miR-146a attenuates lipopolysaccharide (LPS)-induced inflammatory cytokine production in RAW264.7 cells. a Schematic image of the key inflammatory response mediated through the activation of Toll-like receptor and the downstream nuclear factor kappa $B$ (NF-KB) pathway. b, c Concentrations of interleukin (IL)-6 and tumor necrosis factor (TNF)- $\alpha$ in the supernatant of RAW264.7 cells were evaluated by enzyme-linked immunosorbent assay (ELISA) $6 \mathrm{~h}$ after of LPS stimulation. Data are shown as mean \pm standard error of mean (SEM); $n=3$ per group, $* p<0.05$ by one-way ANOVA and following

\section{miR-146a-expressing plasmid improves survival after CLP}

We explored whether miR-146a alters sepsis-induced mortality and organ dysfunction in septic mice using a

Tukey's test. d, e, f Western blot analysis of interleukin 1 receptorassociated kinase 1 (IRAK-1) and tumor necrosis receptor-associated factor 6 (TRAF6) expression in RAW264.7 cells. Data are shown as mean \pm SEM; $n=5$ per group. ${ }^{*} p<0.05$ by one-way ANOVA, and following Tukey's test. $\mathbf{g}, \mathbf{h}$ The transcriptional activity of NF- $\kappa \mathrm{B}$ and AP-1 was significantly attenuated in miR-146a-transfected cells as compared with control cells or negative control-transfected cells after 30 min of LPS stimulation. Data are shown as mean \pm SEM; $n=5$ per group. ${ }^{*} p<0.05$ by one-way ANOVA, and following Tukey's test

miR-146a-expressing plasmid that also encoded EGFP (Fig. S2A). We injected the miR-146a-expressing plasmid/PEI complex into mice via tail veins (miR-146a plasmid group). As a negative control, an empty plasmid/ PEI complex was injected (empty plasmid group) 
A

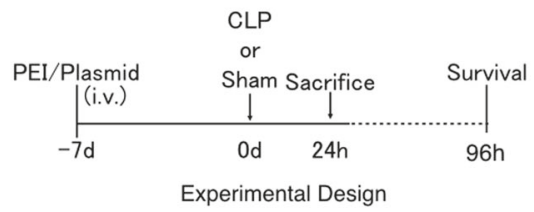

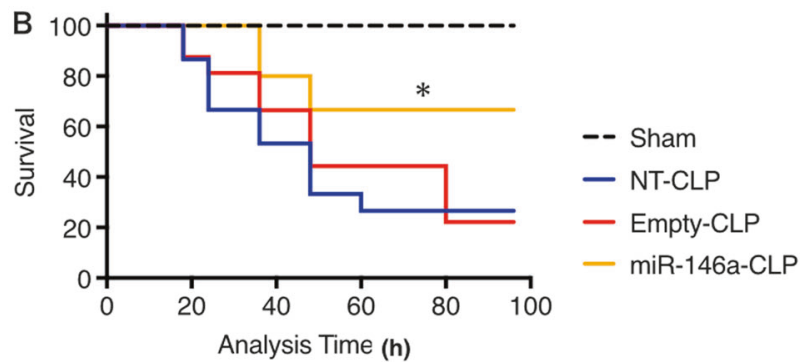
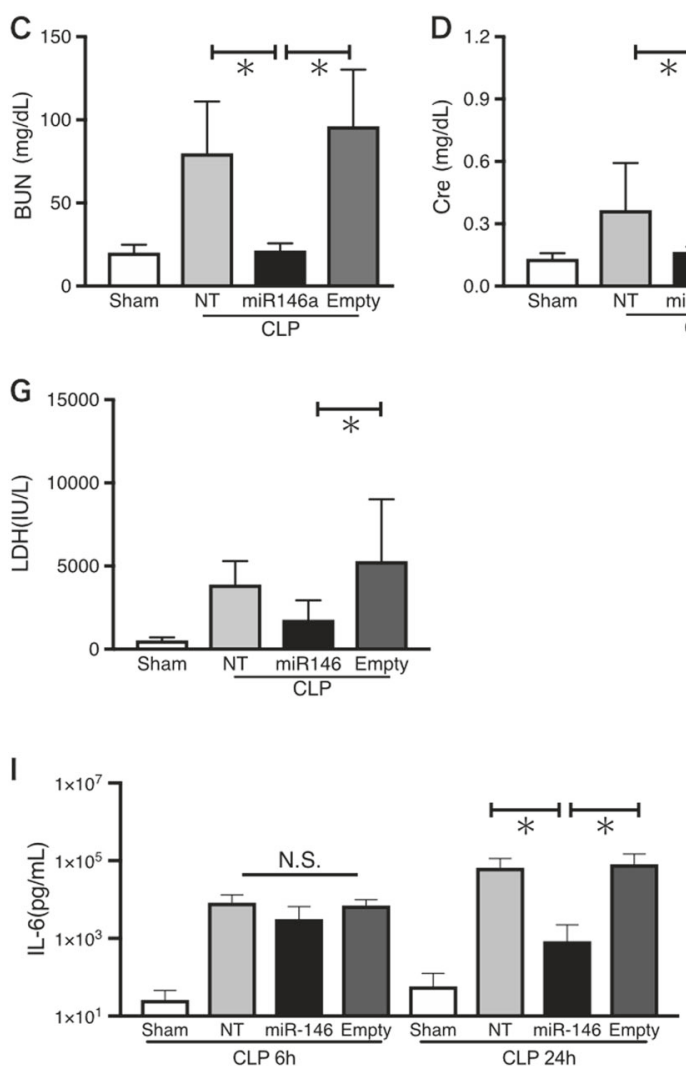

Fig. 2 Induction of miR-146a expression attenuates the severity of cecal ligation and puncture (CLP)-induced polymicrobial sepsis in mice. a Experimental protocol of plasmid injection and sepsis induction. b: Comparison of survival after CLP procedure among mice from the sham-operated, miR-146a-expressing plasmid/PEI-treated, empty plasmid/PEI-treated, and non-treated groups. ( $n=11$, sham; $n=15$, NT-CLP; $n=16$, empty-CLP; $n=15$, miR-146a-CLP; * $p<0.05 \mathrm{miR}-$ 146a-CLP versus empty-CLP or NT-CLP by Log-rank test). $\mathbf{c}-\mathbf{g}$ Concentrations of blood urea nitrogen (BUN), serum creatinine (Cre),

(Fig. S2B). Prior to the induction of sepsis, we checked for organ toxicity in mice injected with plasmid/PEI and found no evidence of renal or liver toxicity (S-Fig. 3A-E). Seven days after the injection, mice were subjected to polymicrobial sepsis via CLP surgery (Fig. 2a). The survival rate was better in the miR-146a group than in the non-treated group and empty plasmid group (Fig. 2b), suggesting that miR-146a may attenuate the severity of CLP-induced sepsis.
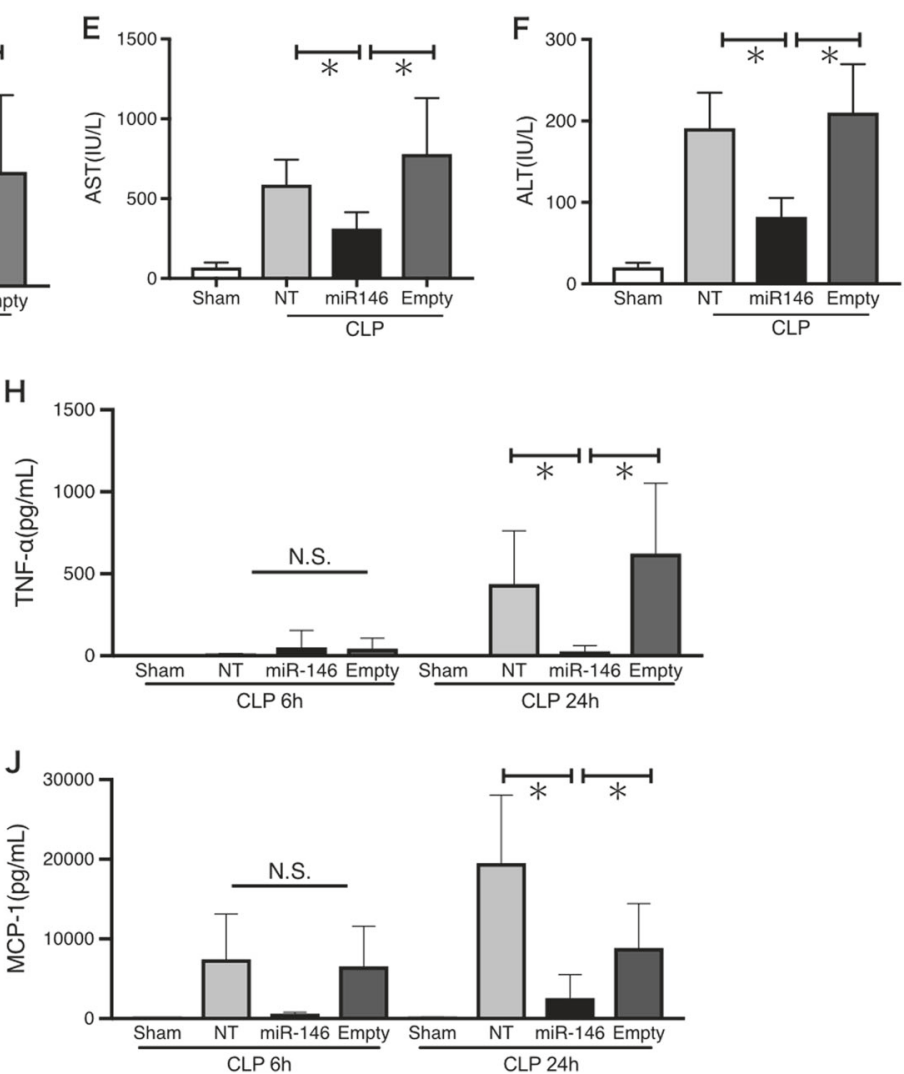

aspartate aminotransferase (AST), alanine aminotransferase (ALT), and lactate dehydrogenase (LDH) at $24 \mathrm{~h}$ after CLP. Data are shown as mean \pm standard error of mean (SEM); $n=6-9$ per group. ${ }^{*} p<0.05$ by one-way ANOVA, and following Tukey's test. $\mathbf{h}-\mathbf{j}$ Levels of serum inflammatory cytokines interleukin (IL)-6, tumor necrosis factor-alpha $(\mathrm{TNF}-\alpha)$, and monocyte chemoattractant protein 1 (MCP-1) as evaluated by enzyme-linked immunosorbent assay (ELISA) at $6 \mathrm{~h}$ and $24 \mathrm{~h}$ after CLP. Data are shown as mean \pm SEM; $n=6-9$ per group. ${ }^{*} p<$ 0.05 by one-way ANOVA and following Tukey's test

\section{miR-146a-expressing plasmid prevents sepsis- induced organ failure and inflammatory cytokine production}

Serum BUN, Cre, AST, ALT, and LDH levels were measured $24 \mathrm{~h}$ after CLP. In the non-treated and empty plasmid groups, these parameters were significantly elevated, consistent with previously reported results [27-29]. However, a significant reduction in these parameters was observed in 
mice treated with miR-146a plasmid (Fig. 2c-g). In addition, serum TNF- $\alpha$, IL- 6 , and MCP-1 levels were measured at $6 \mathrm{~h}$ and $24 \mathrm{~h}$ after CLP. Serum cytokine levels were slightly, but not significantly, elevated in the CLP groups at $6 \mathrm{~h}$. In contrast and consistent with the results of organ damage assessment, the inflammatory cytokine levels were significantly suppressed $24 \mathrm{~h}$ after CLP in the miR-146a plasmid group compared to the non-treated and empty plasmid groups (Fig. $2 \mathrm{~h}-\mathrm{j}$ ). When the plasmid/PEI complex was injected just following the CLP procedure, the inflammatory response was not attenuated in the miR-146a group, which displayed the same poor survival outcome as other groups (Fig. S4A-D).

\section{Plasmid/PEl complex is mainly distributed in splenic macrophages}

To evaluate the distribution of the plasmid/PEI complex, we first performed ex vivo imaging (Fig. 3a) and detected EGFP signal in the lung, liver, kidney, and spleen. The signal was most intense in the spleen. Next, we evaluated EGFP expression in the lung, liver, spleen, and kidney by immunohistochemistry (Fig. 3b). The number of $\mathrm{EGFP}^{+}$ cells was significantly higher in the spleen than in other organs (Fig. 3c). Although we observed localization of $\mathrm{EGFP}^{+}$cells in the liver and lungs, the signal was much weaker than that observed in the spleen. These data suggested that the injected plasmid/PEI complex is mainly distributed in the spleen. Thus, we used spleen samples for further analyses [30].

To confirm the localization of the plasmid in the spleen, we stained splenic tissues with an anti-CD68 antibody and observed the co-localization of EGFP and $\mathrm{CD}^{+} 8^{+}$macrophages (Fig. 4a, b). The similar result was observed in the liver (Fig. S5A, B). Next, we isolated $\mathrm{F} 4 / 80^{+}$cells from splenic cell suspensions and found that miR-146a expression was increased in $\mathrm{F} 4 / 80^{+}$cells (Fig. 4c). In contrast, F4/ $80^{-}$cells failed to show miR-146a overexpression (Fig. 4c). A similar result was observed in $\mathrm{CD} 11 \mathrm{~b}^{+}$cells (Fig. $4 \mathrm{~d}$ ). Additionally, $\mathrm{F} 4 / 80^{+}$cells and $\mathrm{CD} 11 \mathrm{~b}^{+}$cells expressed EGFP in the miR-146a plasmid group and the empty plasmid group (Fig. 4e, f). These data strongly suggested that the plasmid/PEI complex is mainly distributed in splenic macrophages, where it has a functional role.

In addition, we evaluated inflammatory cytokine levels and NF-KB activity in the spleen. IL-6 level was significantly decreased in the miR-146a plasmid group $24 \mathrm{~h}$ after CLP compared to the empty plasmid-treated and non-treated CLP groups (Fig. 5a). In the miR-146a plasmid group, NF-кB activity was attenuated at $6 \mathrm{~h}$ after CLP as compared with the empty plasmid group (Fig. 5b). Next, we performed immunostaining for cleaved caspase-3 and TUNEL staining, and observed fewer cleaved caspase-3 or TUNEL-positive cells in the miR-146a plasmid group than in the empty plasmid and the non-treated CLP groups (Fig. 5c-e). These results indicated that miR-146a-expressing plasmid attenuated the
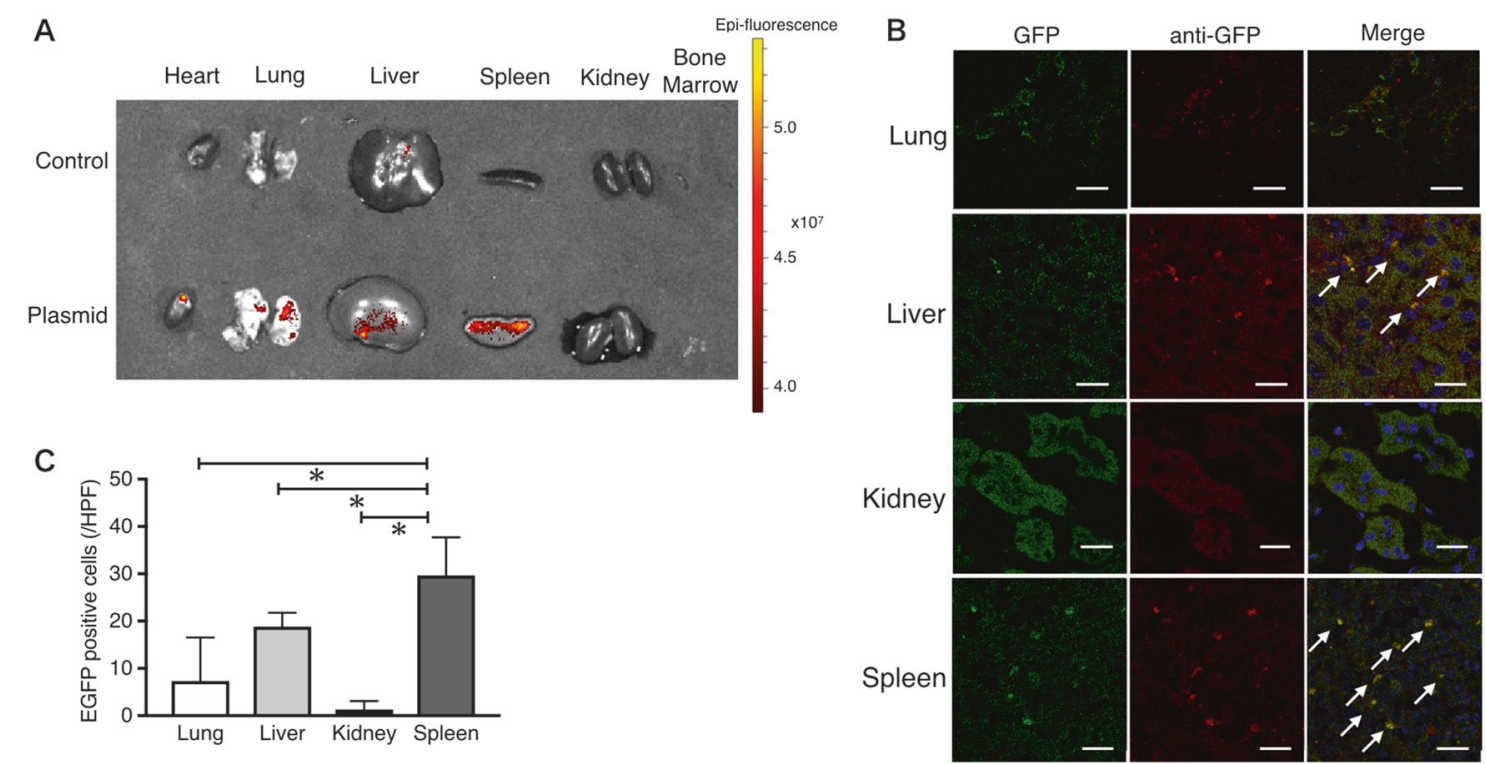

Fig. 3 Injected miR-146a-expressing plasmid/PEI complex was mainly distributed in the spleen. a Comparison of EGFP expression by ex vivo imaging in organs from control mice and mice treated with the $\mathrm{PEI} /$ plasmid complex. Fluorescence intensity is shown as photo$\mathrm{n} \cdot \mathrm{sec} \cdot{ }^{-1} \mathrm{~cm}^{-2}$, and the color of the signal represents the amount of EGFP protein present. b Confocal microscopy images of EGFP in the

lung, liver, kidney, and spleen at day 7 after injection of plasmid/PEI complex. Scale bars represent $100 \mu \mathrm{m}$. c Comparison of the number of EGFP-positive cells in each organ. Data are shown as means \pm standard error of mean (SEM); $n=6$ per group. ${ }^{*} p<0.05$ by one-way ANOVA and Tukey's test 
A
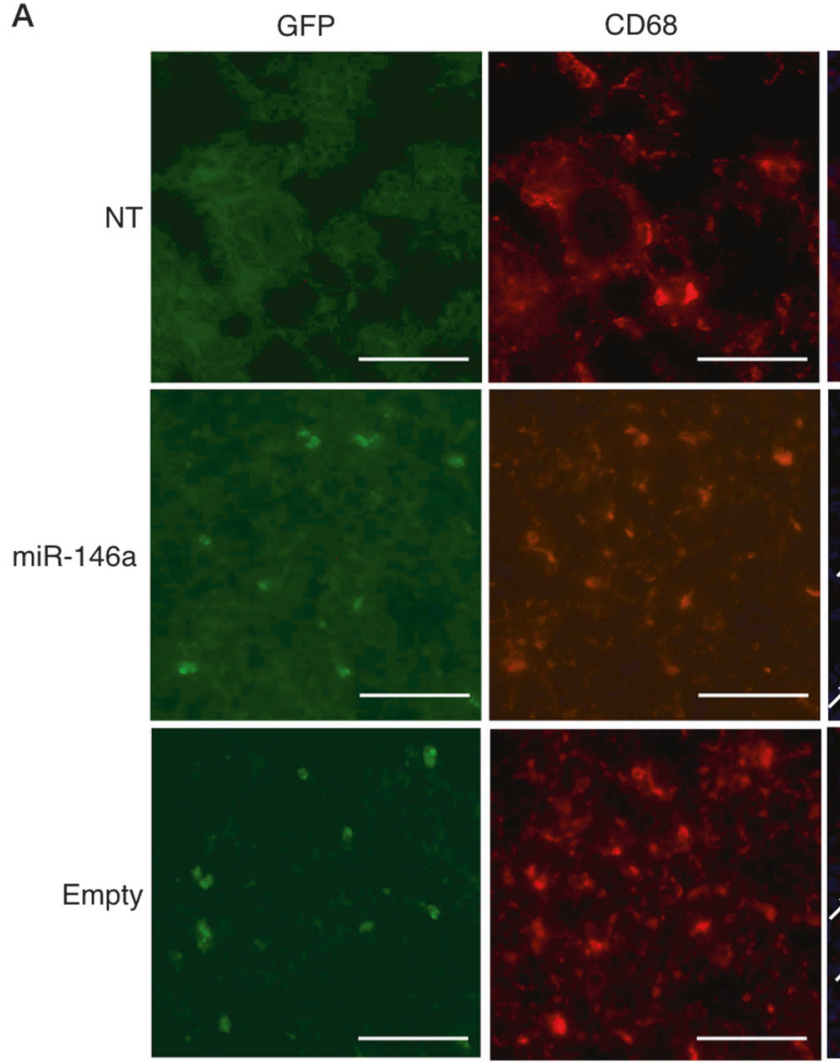

C
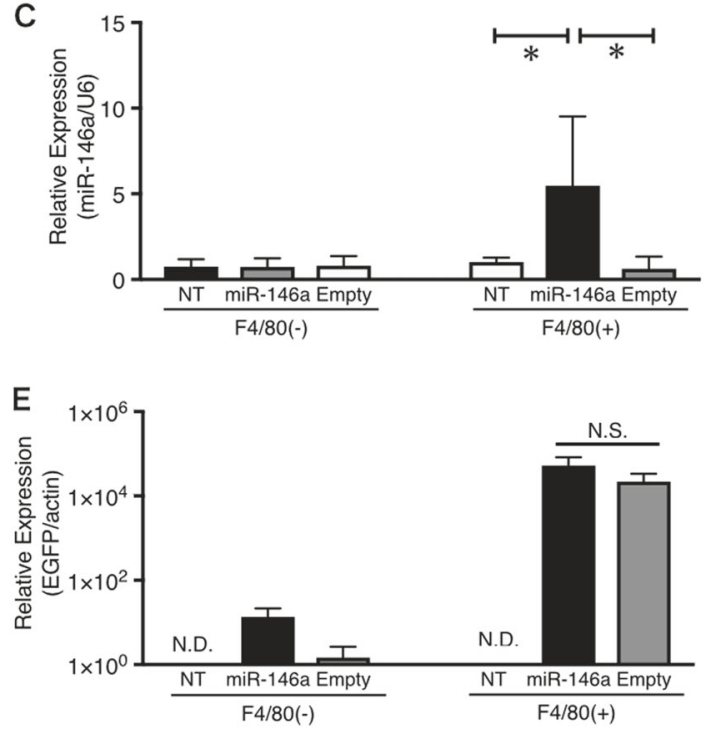

Fig. 4 Macrophage localization of miR-146a-expressing plasmid/PEI complex in the spleen. a Immunohistochemistry of $\mathrm{CD} 68^{+}$macrophages (red) and EGFP (green) in spleens harvested from the plasmid/ PEI complex-injected mice. Scale bars represent $100 \mu \mathrm{m}$. b The number of $\mathrm{EGFP}^{+} \mathrm{CD}^{+}$cells was higher in the plasmid/PEI complex-injected group than in non-treated group. Data are shown as mean \pm standard error of mean (SEM); $n=3$ per group. ${ }^{*} p<0.05$ by
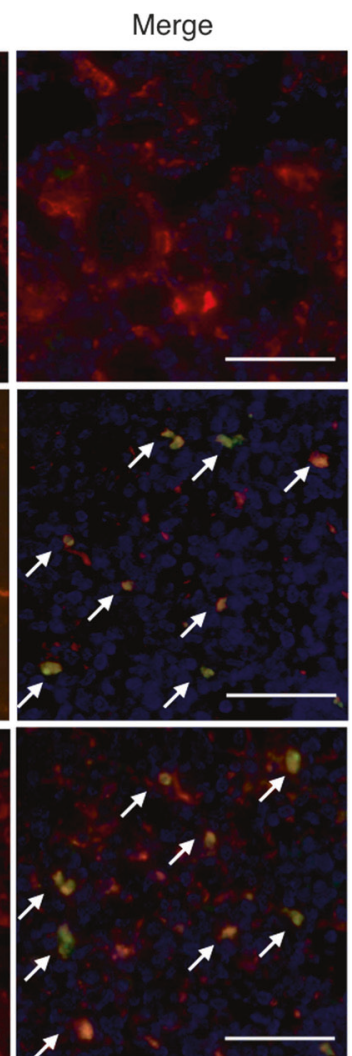

B
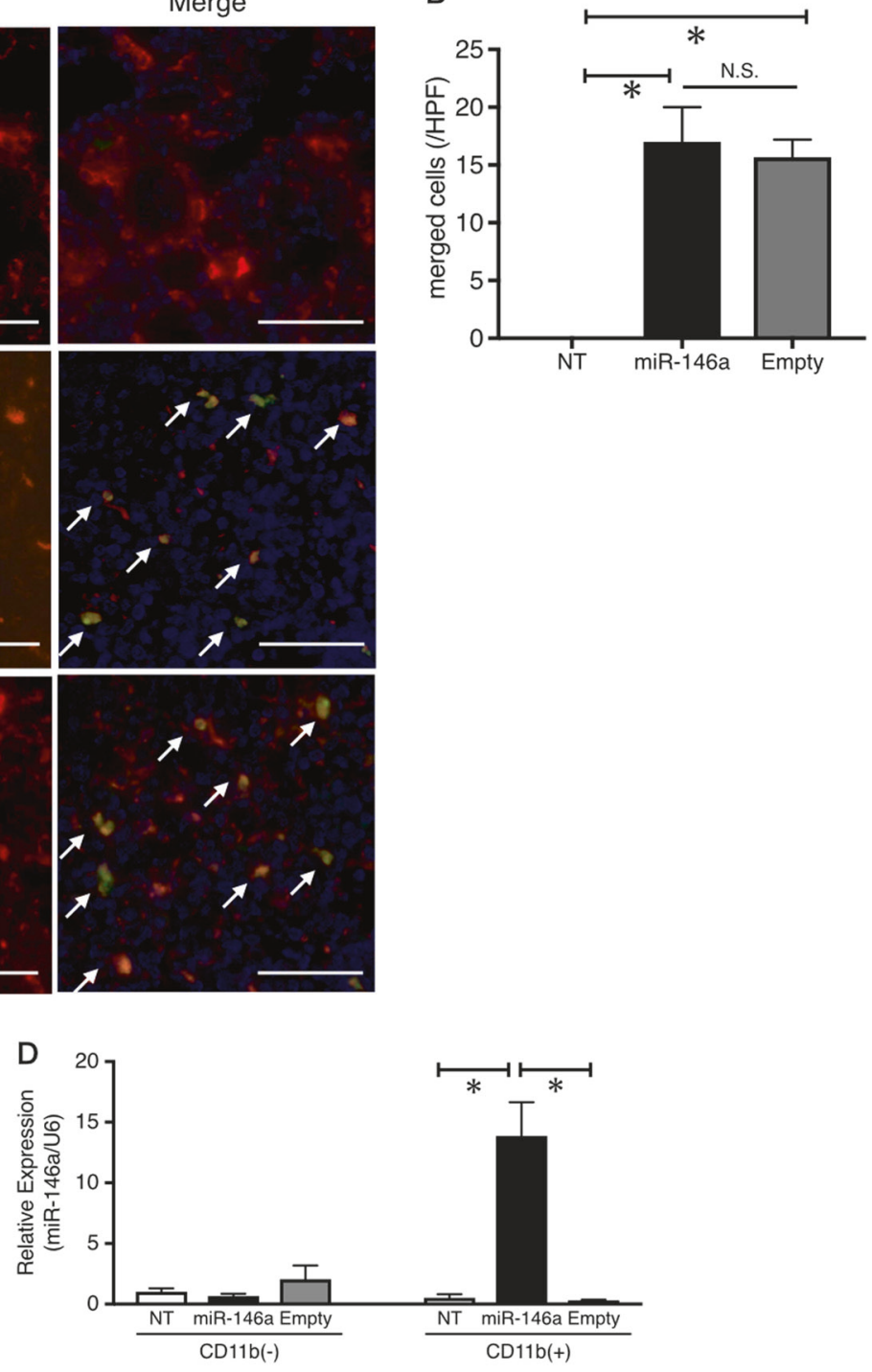

$\mathrm{F}$

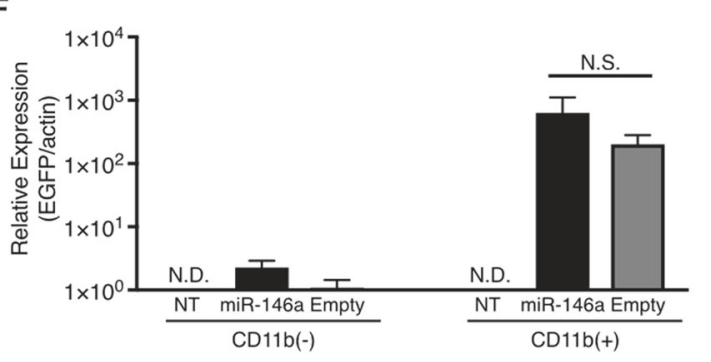

one-way ANOVA and Tukey's test. c Expression of miR-146a in F4/ $80^{+}$cells and $\mathrm{F} 4 / 80^{-}$cells separated from the spleen was examined by quantitative polymerase chain reaction (qPCR). d Expression of miR$146 \mathrm{a}$ in $\mathrm{CD}_{11} \mathrm{~b}^{+}$cells and CD11 $\mathrm{b}^{-}$cells separated from the spleen was examined by qPCR. Data are shown as mean \pm SEM; $n=3-6$ per group. ${ }^{*} p<0.05$ by one-way ANOVA and Tukey's test 

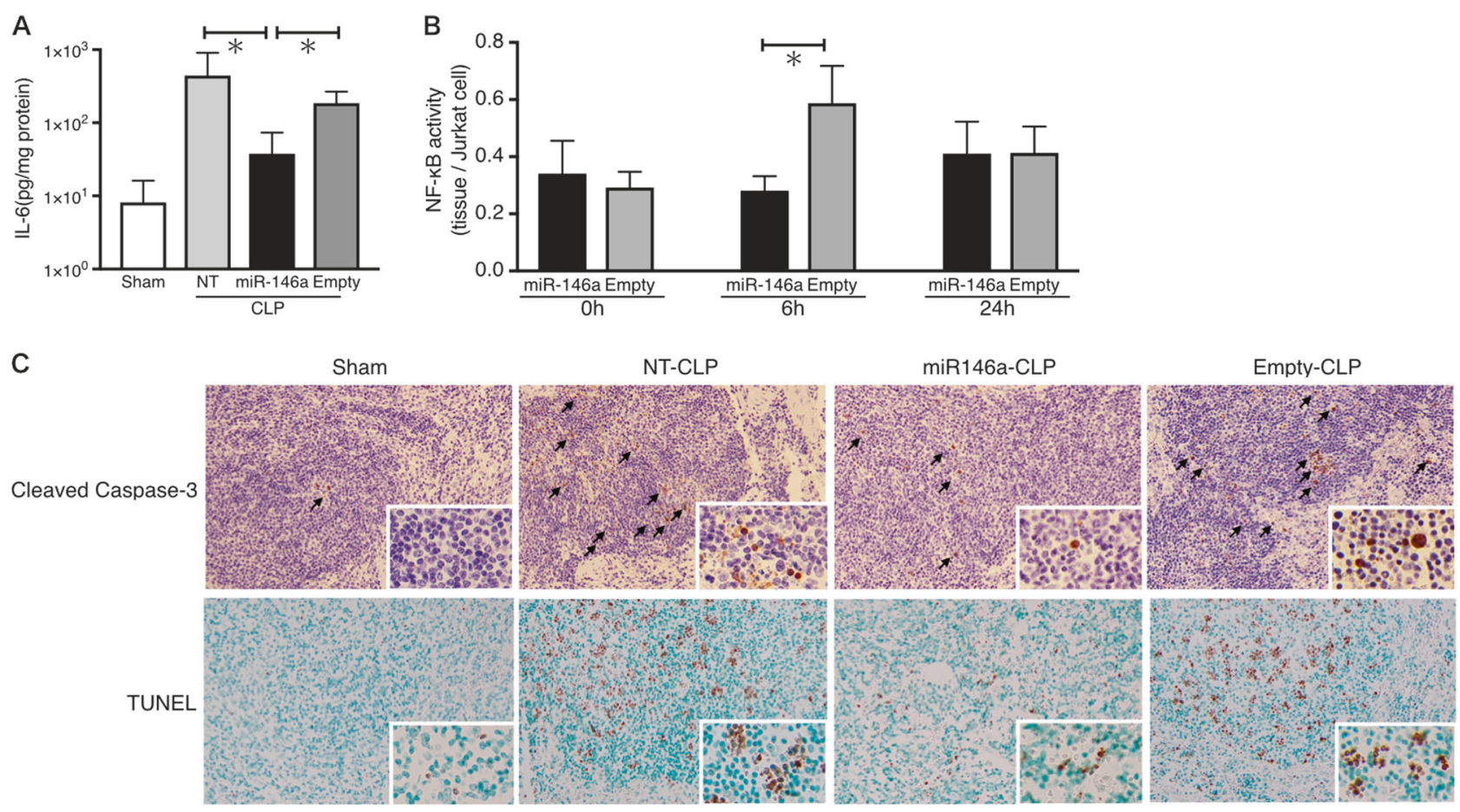

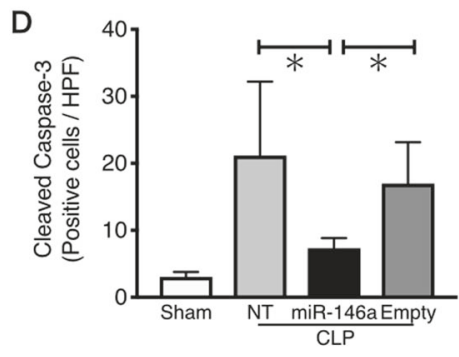

Fig. 5 Treatment with miR-146a-expressing plasmid resulted in antiinflammatory and anti-apoptosis effect in the spleen at $24 \mathrm{~h}$ after cecal ligation and puncture (CLP). Nuclear factor kappa B (NF-kB) activity in the spleen of miR-146a plasmid-treated or empty plasmid-treated mice was evaluated at 0,6 , and $24 \mathrm{~h}$ after CLP. aConcentrations of interleukin (IL)- 6 in the spleen evaluated by enzyme-linked immunosorbent assay (ELISA) at $24 \mathrm{~h}$ after CLP. Data is shown as mean \pm standard error of mean (SEM); $n=6$ per group; $* p<0.05$ one-way ANOVA and Tukey's test. b Transcriptional activity of NF- $\kappa B$ in the

pro-inflammatory pathway but also the pro-apoptotic pathway activated by CLP.

\section{Renal damage induced by sepsis is prevented by miR-146a}

We investigated the effect of miR-146a against sepsisinduced acute kidney injury, which is one of the most frequent and fatal complications in clinical sepsis [2]. Twentyfour $h$ after CLP, renal tubular damage was less severe in the miR-146a plasmid group than in the non-treated CLP and empty plasmid groups (Fig. 6A-a-d, B). In particular, tubular vacuolization, a unique finding in the damaged renal tissue attributed to sepsis, was less prominent in the miR-146a splenic tissue at 0,6 , and $24 \mathrm{~h}$ after CLP. Data are shown as mean \pm SEM; $n=8$ per group. $*^{*} p<0.05$ versus miR-146a plasmid group by Student's $t$-test. c Immunohistochemistry of cleaved caspase-3 expression (upper images) and TUNEL staining (bottom images) in the splenic tissue. Black arrows indicate cleaved caspase-3-positive cells and scale bars represent $100 \mu \mathrm{m}$. d, e Mean value of cleaved caspase-3-positive cells and TUNEL-positive cells in the spleen. Data are shown as mean $\pm \mathrm{SEM} ; n=3-6$ per group. ${ }^{*} p<0.05$ by one-way ANOVA and Tukey's test

plasmid group [31]. In addition, tubular injury was also evaluated by immunostaining of neutrophil gelatinaseassociated lipocalin (NGAL). Fewer NGAL-positive tubules were observed in the miR-146a plasmid group compared with non-treated CLP and empty plasmid groups (Fig. 6A-e-h, C). We also found less $\mathrm{Ly} 6 \mathrm{~B}^{+}$monocyte infiltration in the miR-146a plasmid group than in the nontreated CLP and empty plasmid groups (Fig. 6A-i-1, D).

Consistent with these findings, IL-6 production was decreased in the kidneys of mice from the miR-146a plasmid group (Fig. 6e). EGFP fluorescence and miR-146a overexpression were not observed in any of the groups (Figs. 3B and 6F). Furthermore, the infiltration of Ly6B ${ }^{+}$ macrophages in the liver and lung tissues was less severe in 
A
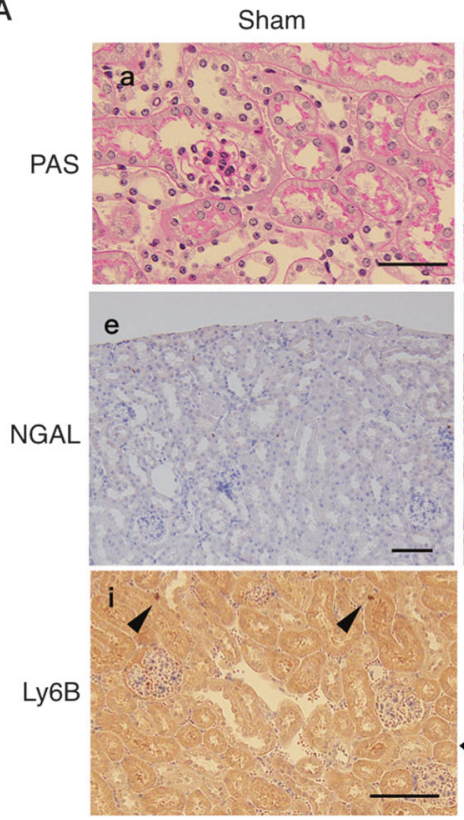

NT-CLP
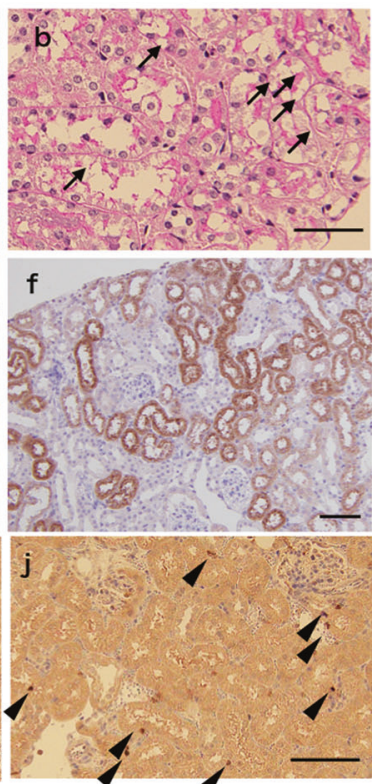

miR146a-CLP
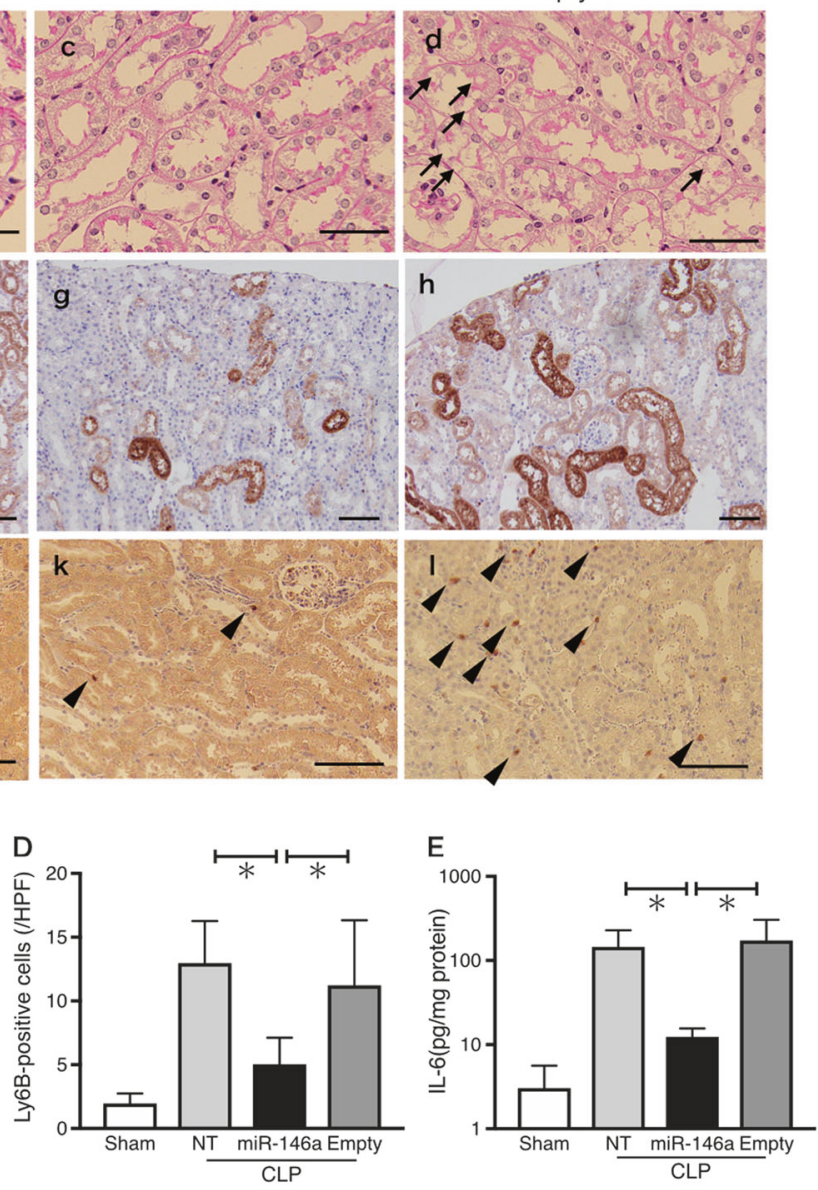

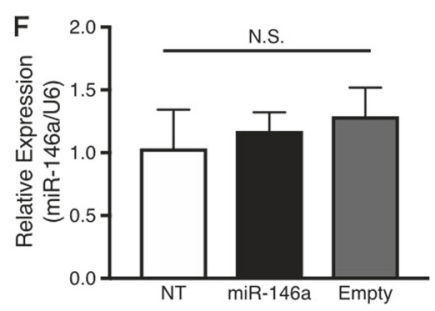

Fig. 6 Treatment with miR-146a-expressing plasmid results in protection from renal damage after cecal ligation and puncture (CLP). After $24 \mathrm{~h}$ of CLP, sepsis-induced renal injury was evaluated. Tubular injury was evaluated as tubular epithelial swelling, loss of brush border, vacuolar degeneration, necrotic tubules, and desquamation. a Periodic acid-Schiff staining (upper images), immunohistochemistry of Ly6B (middle images), and neutrophil gelatinase-associated lipocalin (NGAL; bottom images) for kidney tissues. Arrows in the upper column indicate tubular vacuolization. Arrowheads in the middle column indicate the infiltration of Ly6B-positive macrophages. Scale bars represent $100 \mu \mathrm{m}$. b-d Mean percentage of injured tubules, Ly6B-

the miR-146a group than in the non-treated CLP and empty plasmid groups in spite of the rare EGFP fluorescence (Fig. S6A-D). These results suggested that the attenuation of organ injury, including renal damage after CLP, is caused by the indirect effects of the miR-146a/PEI complex distributed in splenic macrophages. positive macrophages, and NGAL expressing tubules in each group. Data are shown as mean \pm standard error of the mean (SEM); $n=6$ per group. ${ }^{*} p<0.05$ by one-way ANOVA and Tukey's test. e Concentrations of interleukin (IL)-6 in the kidney was evaluated by enzyme-linked immunosorbent assay (ELISA) at $24 \mathrm{~h}$ after CLP. Data are shown as mean $\pm \mathrm{SEM} ; n=6$ per group; $* p<0.05$ versus miR146a plasmid group by one-way ANOVA and Tukey's test. $\mathrm{g}$ Expression of miR-146a, determined by quantitative polymerase chain reaction, failed to increase in the kidney 7 days after the plasmid/PEI injection. Data are shown as mean $\pm \mathrm{SEM} ; n=6$ per group. ${ }^{*} p<0.05$ by one-way ANOVA and Tukey's test

\section{Anti-inflammatory effects of miR-146a are not observed in splenectomized mice}

To evaluate the importance of splenic induction of miR-146a expression in the septic state, we investigated whether miR146a expression plasmid was effective in splenectomized 


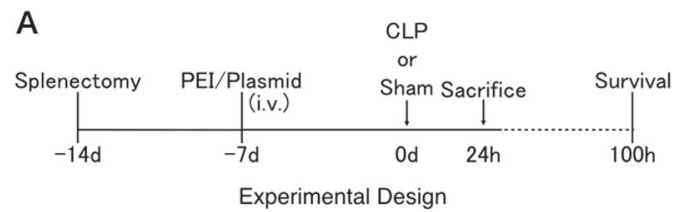

C

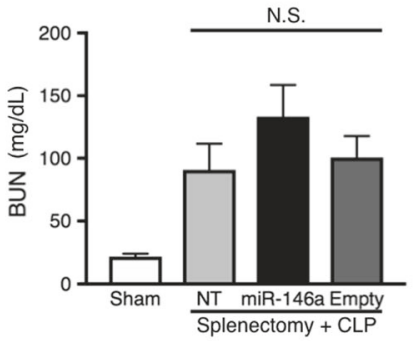

F

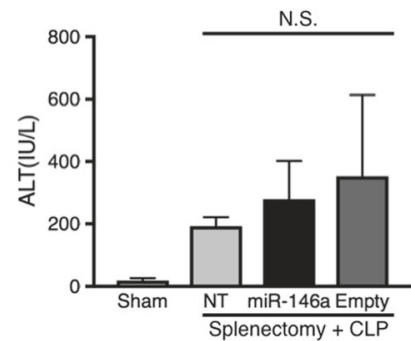

I

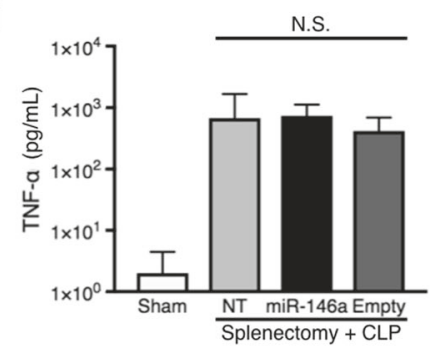

B

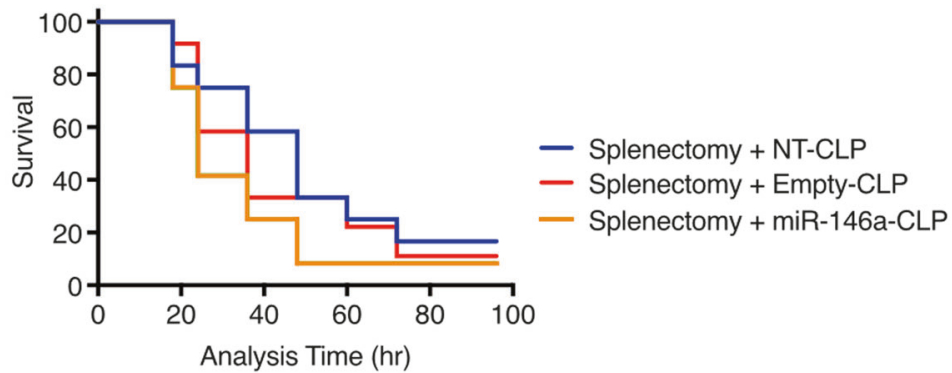

D

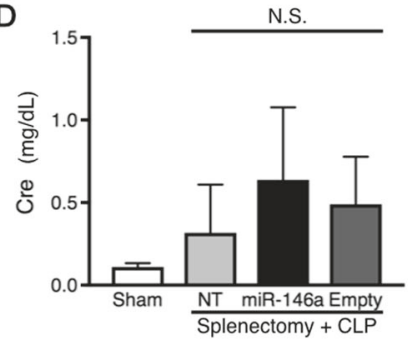

G

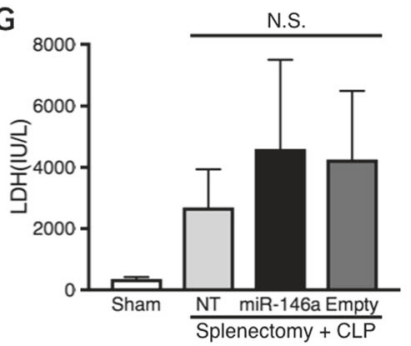

$J$

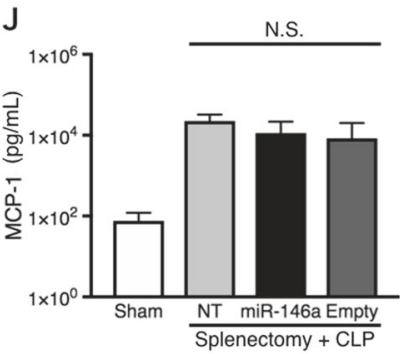

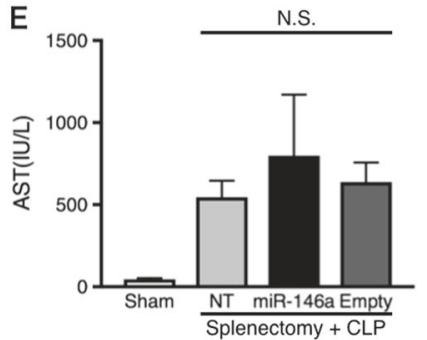

$\mathrm{H}$

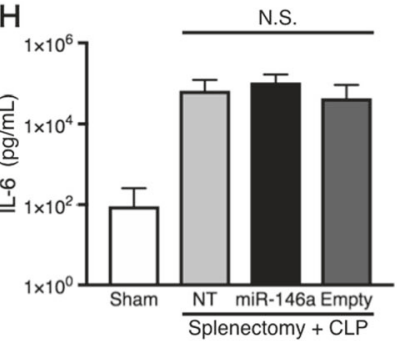

$\mathrm{K}$

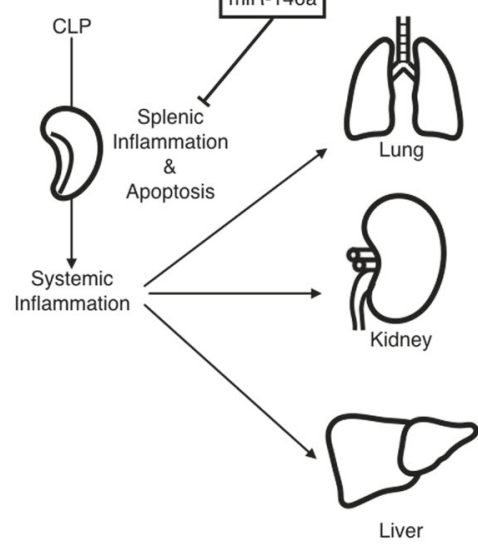

Fig. 7 The anti-inflammatory effect of miR-146a is diminished after splenectomy. a Protocol of cecal ligation and puncture (CLP) for splenectomized mice. b Comparison of survival after splenectomy and CLP procedure. $\mathrm{n}=12$ per group, $\mathrm{p}>0.05$ by Log-rank test. $\mathbf{c}-\mathbf{g}$ Concentrations of serum blood urea nitrogen (BUN), creatinine (Cre), aspartate aminotransferase (AST), alanine aminotransferase (ALT), and lactate dehydrogenase (LDH) at $24 \mathrm{~h}$ after CLP. Data are shown as

mice. Splenectomy was performed 7 days prior to plasmid injection, and the mice were subjected to CLP 7 days after injection (Fig. 7a). The miR-146a-CLP group showed no improvement in survival upon splenectomy (Fig. 7b). mean \pm standard error of mean (SEM); $n=6-9$ per group. $* p<0.05$ by one-way ANOVA, and Tukey's test. h-j Levels of serum inflammatory cytokines, interleukin (IL)-6, tumor necrosis factor-alpha (TNF$\alpha)$, and monocyte chemoattractant protein 1 (MCP-1) at $24 \mathrm{~h}$ after CLP. Data are shown as mean \pm SEM; $n=6-9$ per group. ${ }^{*} p<0.05$ by one-way ANOVA and Tukey's test. $\mathbf{k}$ Schematic image of the miR146a induction strategy in this study

Furthermore, no differences were evident in serum BUN, Cre, AST, ALT, and LDH levels $24 \mathrm{~h}$ after CLP among the splenectomized CLP groups (Fig. 7c-g). In addition, serum levels of IL-6, TNF- $\alpha$, and MCP-1 increased in all 
splenectomized CLP groups (Fig. 7h-j). These data indicated that the miR-146a-PEI injection produces an antiinflammatory effect in the presence of the spleen.

\section{Discussion}

Here, we revealed a novel and critical pathophysiologic role of splenic macrophage interference in sepsis-induced organ injury using the miR-146a-expressing plasmid/PEI complex. The mixture of miR-146a and PEI achieved specific and local transfection of miR-146a into splenic macrophages and led to the attenuation of sepsis-induced cytokine storm, mortality, and multiple organ injury, including acute kidney injury (Fig. 7k).

In addition, we revealed the possible mechanisms underlying the benefit of miR-146a in sepsis-induced organ injury. Signals from TLRs, such as TLR4, activate the NF-KB pathway as part of the complex intracellular signal transduction that occurs in the pathogenesis of sepsis [32]. Presently, miR-146a modulated the transcriptional activity of NF- $\kappa$ B by regulating IRAK-1 and TRAF6 (Fig. 1d-g). Thus, miR-146a has an anti-inflammatory role by regulating NF- $\mathrm{\kappa B}$. Consequently, our results indicate that miR-146a attenuates NF-kBmediated excessive inflammation due to sepsis.

Improvement in the outcomes for patients with sepsis necessitates a firm understanding of the pathophysiology involved and requires the discovery of novel drug targets [33]. In this context, this study provides three important findings. First, the spleen, particularly splenic macrophages, is the key component that governs sepsis-induced multiple organ injury. Following PEI administration, we observed a strong distribution of miR-146a-expressing plasmid in the spleen, while immune responses were strikingly suppressed in many organs, including the kidney, liver, lung, and spleen. Our findings are supported by previous reports of the copious production of cytokines by the reticuloendothelial system, with a response that develops earlier in the spleen than in other organs [27, 34]. The spleen is, therefore, one of the most crucial organs among the mononuclear phagocyte systems [35]. It is conceivable that the main target of the miR-146a-expressing plasmid/PEI complex was the spleen, resulting in the efficient suppression of the cytokine storm (Fig. 7k). No immunomodulation agents (including gene therapy) against sepsis-induced organ injury are currently available. Hence, our strategy to treat splenic macrophages with specific miRNA may serve as a novel therapeutic option in the attenuation of the pathogenesis of sepsis-induced organ injury, including acute kidney injury [36, 37].

Second, this study reveals that miR-146a induction may be a potent therapeutic option for the treatment of sepsisinduced multiple organ injury. We identified a number of
miRNAs as possible therapeutic agents (summarized in Fig. 1a), and found that miR-146a exhibited the strongest effect in the suppression of LPS-induced immune responses. The data provide an insight into the mechanisms underlying sepsis-induced multiple organ injury. The understanding of the exact pathogenesis of sepsis is still incomplete. Sepsis-induced death is hypothesized to be the result of either persistent immune activation with accompanying inflammation and organ failure or immunosuppression and secondary infection during the late phase of sepsis [4]. In the latter situation, immunosuppression is thought to be caused by apoptosis and depletion of immune cells $[38,39]$. In the present study, while miR-146a itself had an immunosuppressive effect, it definitely attenuated the apoptosis of splenocytes, which may mean miR-146a maintained the spleen in a healthy condition. This observation was consistent with previous reports, wherein severe sepsis patients showed apoptosis of splenic cells in clinic and miR-146a showed anti-apoptosis effects in experimental settings [40-42]. We observed that the activity of AP-1, which is a pro-apoptotic signal, was significantly reduced in the spleen after CLP. The observations may partly explain the mechanism of protection of the miR-146a plasmid group from splenic apoptosis. We speculate that the induction of miR-146a in the spleen may be beneficial effects in both the early and late phases of sepsis.

Third, this study raises the possibility of an intriguing therapeutic strategy for sepsis through the combination of miR-146a and PEI. Although investigations into new miRNA agents for clinical use have made great progress, no clinical trials for the treatment of sepsis with miRNA agents have been done [43-45]. A miR-146a-expressing plasmid was shown to improve sepsis-related mortality rates and the severity of multiple organ injury. In addition, we revealed that the major distribution site of the plasmid/ PEI complex was the spleen and that miR-146a overexpression and EGFP signal were rarely observed in the kidney, lung, liver, and bone marrow. The spleen plays a defensive role against infection, but an excessive immune response in the spleen induces the systemic inflammation and multiple organ failure that are the hallmarks of sepsis. In the present study, exogenous miR-146a transfected into the spleen likely regulated the CLP-induced immune response in mice and prevented lethal sepsis. Furthermore, our data suggest that PEI may be a useful material for the delivery of therapeutic agents to splenic macrophages. We also found that the beneficial effects of miR146a were absent in splenectomized mice. This is likely attributed to the attenuated effect of miR-146a following the removal of its main target, the spleen. This observation provides the evidence that the injected plasmid worked in the spleen and was responsible for the suppression of the cytokine storm. 
There are some limitations of the present study. First, the injected plasmid/PEI complex could not improve the outcome if injected after the onset of sepsis (Fig. S4). That may have been because of the requirement of the initial duration for transcription and maturation of the miRNA. Thus, the plasmid/PEI complex could not act immediately. Further studies are needed to establish a strategy, wherein the treatment inception occurs after the onset of sepsis. In addition, there is a possibility that splenectomy itself increased the severity of sepsis [46]. Therefore, the effect of miR-146a was impaired.

In conclusion, we demonstrate that miR-146a induction in splenic macrophages leads to the attenuation of sepsis-induced excessive inflammation, mortality rate, and severity of organ injury. The findings established a novel pathophysiologic role for splenic macrophage interference in sepsis-related organ injury, by using a specific combination of miRNA and PEI-based drug delivery system.

Acknowledgements We thank Norihiko Suzuki, Naoko Asano, and Yuriko Sawa for their excellent technical assistance. We thank Editage (www.editage.jp) for the editing of the manuscript.

\section{Compliance with ethical standards}

Conflict of interest This work was supported in part by a Grant-inAids for Scientific Research (16K09610) and a 2015 research grant from the Aichi Kidney Foundation. Funders had no role in this study.

Publisher's note: Springer Nature remains neutral with regard to jurisdictional claims in published maps and institutional affiliations.

\section{References}

1. Singer M, Deutschman CS, Seymour CW, Shankar-Hari M, Annane D, Bauer M, et al. The Third International Consensus Definitions for Sepsis and Septic Shock (Sepsis-3). JAMA. 2016;315:801-10.

2. Angus DC, Linde-Zwirble WT, Lidicker J, Clermont G, Carcillo J, Pinsky MR. Epidemiology of severe sepsis in the United States: analysis of incidence, outcome, and associated costs of care. Crit Care Med. 2001;29:1303-10.

3. Gigliotti JC, Okusa MD. The spleen: the forgotten organ in acute kidney injury of critical illness. Nephron Clin Pract. 2014;127:153-7.

4. Hotchkiss RS, Monneret G, Payen D. Sepsis-induced immunosuppression: from cellular dysfunctions to immunotherapy. Nat Rev Immunol. 2013;13:862-74.

5. Williams DL, Ha T, Li C, Kalbfleisch JH, Schweitzer J, Vogt $\mathrm{W}$, et al. Modulation of tissue Toll-like receptor 2 and 4 during the early phases of polymicrobial sepsis correlates with mortality. Crit Care Med. 2003;31:1808-18.

6. Gao M, Ha T, Zhang X, Liu L, Wang X, Kelley J, et al. Toll-like receptor 3 plays a central role in cardiac dysfunction during polymicrobial sepsis. Crit Care Med. 2012;40:2390-9.

7. Matsuda N, Hattori Y, Takahashi Y, Nishihira J, Jesmin S, Kobayashi M, et al. Therapeutic effect of in vivo transfection of transcription factor decoy to NF-kappaB on septic lung in mice. Am J Physiol Lung Cell Mol Physiol. 2004;287:L1248-55.

8. Fenhammar J, Rundgren M, Hultenby K, Forestier J, Taavo M, Kenne E, et al. Renal effects of treatment with a TLR4 inhibitor in conscious septic sheep. Crit Care. 2014;18:488.

9. Akira S, Takeda K. Toll-like receptor signalling. Nat Rev Immunol. 2004;4:499-511.

10. Carthew RW, Sontheimer EJ. Origins and mechanisms of miRNAs and siRNAs. Cell. 2009;136:642-55.

11. Li D, Wang A, Liu X, Meisgen F, Grunler J, Botusan IR, et al. MicroRNA-132 enhances transition from inflammation to proliferation during wound healing. J Clin Invest. 2015;125:3008-26.

12. Medina PP, Nolde M, Slack FJ. OncomiR addiction in an in vivo model of microRNA-21-induced pre-B-cell lymphoma. Nature. 2010;467:86-90.

13. Christopher AF, Kaur RP, Kaur G, Kaur A, Gupta V, Bansal P. MicroRNA therapeutics: discovering novel targets and developing specific therapy. Perspect Clin Res. 2016;7:68-74.

14. Bhaumik D, Scott GK, Schokrpur S, Patil CK, Campisi J, Benz CC, et al. Expression of microRNA-146 suppresses NF-kappaB activity with reduction of metastatic potential in breast cancer cells. Oncogene. 2008;27:5643-7.

15. Hou J, Wang P, Lin L, Liu X, Ma F, An H, et al. MicroRNA-146a feedback inhibits RIG-I-dependent Type I IFN production in macrophages by targeting TRAF6, IRAK1, and IRAK2. J Immunol. 2009;183:2150-8.

16. Nahid MA, Pauley KM, Satoh M, Chan EK, et al. miR-146a is critical for endotoxin-induced tolerance: Implication in innate immunity. J Biol Chem. 2009;284:34590-9.

17. Albers J, Rajski M, Schonenberger D, Harlander S, Schraml P, von Teichman A, et al. Combined mutation of Vhl and Trp53 causes renal cysts and tumours in mice. EMBO Mol Med. 2013;5:949-64.

18. Matsuda N, Hattori Y, Jesmin S, Gando S, et al. Nuclear factorkappaB decoy oligodeoxynucleotides prevent acute lung injury in mice with cecal ligation and puncture-induced sepsis. Mol Pharmacol. 2005;67:1018-25.

19. Doi K, Hu X, Yuen PS, Leelahavanichkul A, Yasuda H, Kim SM, et al. AP214, an analogue of alpha-melanocyte-stimulating hormone, ameliorates sepsis-induced acute kidney injury and mortality. Kidney Int. 2008;73:1266-74.

20. Morishita Y, Imai T, Yoshizawa H, Watanabe M, Ishibashi K, Muto S, et al. Delivery of microRNA-146a with polyethylenimine nanoparticles inhibits renal fibrosis in vivo. Int $\mathrm{J}$ Nanomed. 2015;10:3475-88.

21. Kato N, Yuzawa Y, Kosugi T, Hobo A, Sato W, Miwa Y, et al. The E-selectin ligand basigin/CD147 is responsible for neutrophil recruitment in renal ischemia/reperfusion. J Am Soc Nephrol. 2009;20:1565-76.

22. Leelahavanichkul A, Yasuda H, Doi K, Hu X, Zhou H, Yuen PS, et al. Methyl-2-acetamidoacrylate, an ethyl pyruvate analog, decreases sepsis-induced acute kidney injury in mice. Am J Physiol Ren Physiol. 2008;295:F1825-35.

23. Essandoh K, Fan GC. Role of extracellular and intracellular microRNAs in sepsis. Biochim Biophys Acta. 2014;1842: 2155-62.

24. Li T, Morgan MJ, Choksi S, Zhang Y, Kim YS, Liu ZG. MicroRNAs modulate the noncanonical transcription factor NFkappaB pathway by regulating expression of the kinase IKKalpha during macrophage differentiation. Nat Immunol. 2010; 11:799-805.

25. Feng X, Wang H, Ye S, Guan J, Tan W, Cheng S, et al. Upregulation of microRNA-126 may contribute to pathogenesis of ulcerative colitis via regulating NF-kappaB inhibitor IкB $\alpha$. PLoS ONE. 2012;7:e52782. 
26. Wendlandt EB, Graff JW, Gioannini TL, McCaffrey AP, Wilson ME. The role of microRNAs miR-200b and miR-200c in TLR4 signaling and NF-kappaB activation. Innate Immun. 2012;18:846-55.

27. Yasuda H, Leelahavanichkul A, Tsunoda S, Dear JW, Takahashi Y, Ito S, et al. Chloroquine and inhibition of Toll-like receptor 9 protect from sepsis-induced acute kidney injury. Am J Physiol Ren Physiol. 2008;294:F1050-58.

28. Miyaji T, Hu X, Yuen PS, Muramatsu Y, Iyer S, Hewitt SM, et al. Ethyl pyruvate decreases sepsis-induced acute renal failure and multiple organ damage in aged mice. Kidney Int. 2003;64:1620-31.

29. Luo CJ, Luo F, Zhang L, Xu Y, Cai GY, Fu B, et al. Knockout of interleukin-17A protects against sepsis-associated acute kidney injury. Ann Intensive Care. 2016;6:56.

30. Malek A, Merkel O, Fink L, Czubayko F, Kissel T, Aigner A. In vivo pharmacokinetics, tissue distribution and underlying mechanisms of various PEI(-PEG)/siRNA complexes. Toxicol Appl Pharmacol. 2009;236:97-108.

31. Doi K, Leelahavanichkul A, Yuen PS, Star RA. Animal models of sepsis and sepsis-induced kidney injury. J Clin Invest. 2009;119:2868-78.

32. Hattori Y, Hattori K, Suzuki T, Palikhe S, Matsuda N. Nucleicacid based gene therapy approaches for sepsis. Eur J Pharmacol. 2018;833:403-10.

33. Doi K. Role of kidney injury in sepsis. J Intensive Care. 2016;4:17.

34. Hadjiminas DJ, McMasters KM, Peyton JC, Cheadle WG. Tissue tumor necrosis factor mRNA expression following cecal ligation and puncture or intraperitoneal injection of endotoxin. J Surg Res. 1994;56:549-55.

35. Thompson PN, Cho E, Blumenstock FA, Shah DM, Saba TM. Liver and spleen phagocytic depression after peripheral ischemia and reperfusion. Am J Surg. 1992;164:248-53.

36. Marshall JC. Why have clinical trials in sepsis failed? Trends Mol Med. 2014;20:195-203.
37. Jo SK, Rosner MH, Okusa MD. Pharmacologic treatment of acute kidney injury: why drugs haven't worked and what is on the horizon. Clin J Am Soc Nephrol. 2007;2:356-65.

38. Hotchkiss RS, Karl IE. The pathophysiology and treatment of sepsis. N Engl J Med. 2003;348:138-50.

39. Hotchkiss RS, Swanson PE, Freeman BD, Tinsley KW, Cobb JP, Matuschak GM, et al. Apoptotic cell death in patients with sepsis, shock, and multiple organ dysfunction. Crit Care Med. 1999;27:1230-51.

40. Huang W, Tian SS, Hang PZ, Sun C, Guo J, Du ZM. Combination of microRNA-21 and microRNA-146a attenuates cardiac dysfunction and apoptosis during acute myocardial infarction in mice. Mol Ther Nucleic Acids. 2016;5:e296.

41. Deng S, Wang H, Jia C, Zhu S, Chu X, Ma Q, et al. MicroRNA146a induces lineage-negative bone marrow cell apoptosis and senescence by targeting polo-like kinase 2 expression. Arterioscler Thromb Vasc Biol. 2017;37:280-90.

42. Hotchkiss RS, Monneret G, Payen D. Sepsis-induced immunosuppression: from cellular dysfunctions to immunotherapy. Nat Rev Immunol. 2013;13:862-74.

43. Janssen HL, Reesink HW, Lawitz EJ, Zeuzem S, RodriguezTorres M, Patel K, et al. Treatment of HCV infection by targeting microRNA. N Engl J Med. 2013;368:1685-94.

44. A Multicenter Phase I Study of MRX34, MicroRNA miR-RX34 Liposomal Injection. [Internet], Clinical Trials. gov. A service of the U.S. National Institutes of Health. https://clinicaltrials.gov/ct2/ show/NCT01829971. Accessed 15 Aug 2017.

45. Natural History of Disease Study in Alport Syndrome Patients (RG012-01). [Internet], Clinical Trials. gov. A service of the U.S. National Institutes of Health. https://clinicaltrials.gov/ct2/show/ NCT02136862?term $=$ RG012\&recrs $=a \&$ rank $=1$. Accessed 14 Aug 2017.

46. Krivit W. Overwhelming postsplenectomy infection. Am J Hematol. 1977;2:193-201. 